\title{
SDSS-based taxonomic classification and orbital distribution of main belt asteroids
}

\author{
J. M. Carvano ${ }^{1}$, P. H. Hasselmann ${ }^{1,2}$, D. Lazzaro ${ }^{1}$, and T. Mothé-Diniz ${ }^{2}$ \\ 1 Observatório Nacional (COAA), rua Gal. José Cristino 77, São Cristóvão, CEP 20921-400 Rio de Janeiro RJ, Brazil \\ e-mail: carvano@on.br \\ ${ }^{2}$ Universidade Federal do Rio de Janeiro/Observatório do Valongo, Lad.Pedro Antônio, 43, 20080-090 Rio de Janeiro, Brazil
}

Received 18 September 2009 / Accepted 4 November 2009

\begin{abstract}
Aims. The present paper aims to derive a new classification scheme for SDSS MOC asteroid colors that is compatible with previous taxonomies based on spectroscopic data. The distribution of these can give important clues to the formation and evolution of this region of the Solar System, as well as to locate candidates with mineralogically interesting spectra for detailed observations.

Methods. The methodology is based on the large database SDSS MOC4. Templates of the main taxonomic classes are derived and then used to classify the asteroid observations in the SDSS MOC4. The derived taxonomic scheme is compatible with the Bus taxonomy and is suitable to the peculiarities of the SDSS observations, in particular, the low spectral resolution.

Results. Density maps of the seven classes defined by the method reproduce classical results for the background which is mainly dominated by the $S_{\mathrm{p}}$ class in the inner belt and by the $X_{\mathrm{p}}$ and the $C_{\mathrm{p}}$ classes beyond $2.8 \mathrm{AU}$. It also shows new structures, such as the fact that the $X_{\mathrm{p}}$ and $C_{\mathrm{p}}$ seem evenly distributed in the inner belt while in the outer belt the $S_{\mathrm{p}}$ class increase in density only at the location of asteroid families. Although their overall distribution is similar, the $X_{\mathrm{p}}$ class seems less frequently associated with large families than do the $C_{\mathrm{p}}$ class asteroids. Although only clustering around the Vesta family, the $V_{\mathrm{p}}$ class asteroidsnappear scattered all around the main belt. Besides the lack satisfactory explanations of most of the highlighted features, they may provide strong constraints on the models of the formation and evolution of the Solar System.
\end{abstract}

Key words. minor planets, asteroids: general - methods: miscellaneous

\section{Introduction}

Since the early works in asteroid taxonomy, there has been a tendency to establish firm relations between the derived taxonomic classes and mineralogy. At that time, the only analytical tool available to researchers were direct comparison between the visible reflectance spectra of asteroids and those of meteorite and mineral samples obtained in laboratory. Indeed, by using such methodology McCord et al. (1970) were able to establish a very robust relation between the asteroid (4) Vesta and the howardite, eucrite, and diogenite (HED) meteorites. Possibly inspired by this accomplishment, in the early taxonomies (Chapman et al. 1975; Bowell et al. 1978) the classes were denominated by letters according to the mineralogical interpretation of their observational properties. In this way, the $\mathrm{C}$ class was associated to carbonaceous chondrites, the $\mathrm{S}$ class to stony-iron meteorites, the $\mathrm{E}$ class to enstatite achondrites, and the $M$ class to the metallic iron-nickel meteorites. Later taxonomies (Tholen 1984; Barucci et al. 1987; Tedesco et al. 1989; Bus \& Binzel 2002a) kept most of the class denominations. With the increase in the number of observations available and the improvement in classification algorithms, the number of classes increased between the first and the second generation of taxonomies, culminating in the Bus taxonomy (Bus \& Binzel 2002a), which defines 26 classes.

The direct mineralogical characterization of the asteroids, however, has proved to be much harder to achieve. It is severely complicated by (Gaffey et al. 2002): 1) the existence of several factors (surface texture, observation geometry, space weathering effects) that are not directly related to mineralogy but may affects the observed reflectance spectra of asteroids; 2) the fact that not all mineralogical assemblages exhibit diagnostic features in their spectra; and 3) most of the diagnostic spectral bands of the most abundant minerals tend to be broad and to overlap heavily. Despite the difficulties, considerable progress has been made in this area, some of which have shown how uncertain the mineralogical inferences made from taxonomy can be. A good example here is the discovery that some E- and M-class asteroids show hydration bands around $3 \mu \mathrm{m}$ (Jones et al. 1990; Rivkin et al. 1995, 2000). The presence of this band indicate that the asteroids did not suffer severe heating during their evolution, which is clearly inconsistent with the thermal history of enstatite achondrites and nickel-iron meteorites. In the face of the evolution of our understanding of the mineralogy of asteroids, it is becoming evident that taxonomy alone is a poor tool for infering composition.

These limitations do not mean, however, that asteroid taxonomies are of no use. The fact that some classes have been, with minor modifications, systematically recovered by independent authors using diverse data sets and different methodologies suggests that they convey very real information about the diversity of spectral properties of asteroids. Taxonomic classification is paramount to selecting targets for detailed investigation. Also, given the scarcity of reliable mineralogic characterization of asteroids, the use of taxonomic classes is still the best available 
way to study the large-scale distribution of compositions in the main asteroid belt. And, although taxonomy alone is not sufficient to make meaningful mineralogical inferences, the analysis of the classes distribution in the main belt can, at least, highlight regions and objects that deserve further investigation.

Given these two main applications of taxonomy - target selection and statistical studies - it is fair to say that the usefulness of a given taxonomic scheme is proportional to the number of objects it potentially can classify. This depends on several factors, among which are the number of suitable observations that are available at any given time. The Bus taxonomy represented an increase of about an order of magnitude over early efforts. Here taxonomies based on data of large photometric surveys, like the Sloan Digital Sky Survey (SDSS), offer a clear advantage, since they can provide observations for much more objects than what can be reasonably obtained from spectroscopy. Several works have been using taxonomy classification inferred from the SDSS colors, but so far without formal linkage to previous taxonomies. Such work, however, is needed to provide a robust link to previous studies that use those taxonomies. Also, it will be useful as a basis of comparison to the next logical step, which is to derive an independent taxonomic system based on the SDSS data, which may reveal new putative spectral classes that could have eluded previous efforts based on smaller datasets.

In the present work we define a classification algorithm based on the SDSS colors. It seeks to be compatible with previous taxonomic schemes based on spectroscopic data. The goal here is not to define a new taxonomy, but merely to provide a concise method of assigning taxonomic classes to SDSS observations of asteroids that are compatible with the limitations of the dataset. This scheme is then applied to the asteroids listed in the fourth release of the SDSS Moving Object Catalogue (MOC4) and to meteorite spectra from the RELAB database. These results are used to analyze the distributions of taxonomies in the main asteroid belt. We also use the large number of asteroids that were observed multiple times in the MOC4 to analyze the issue of taxonomic variations among observations. Since there are very few definite conclusions about mineralogy that can be drawn based solely on taxonomy, the ultimate goal here is to identify interesting targets for NIR spectroscopic followup observations and to study the distribution of taxonomies in families and in the main belt as a whole.

\section{Methodology}

\subsection{Parameterization of the observations}

The definition of a taxonomic scheme depends on the choice of the observational parameters and of the methodology used for separating the observations among classes. The Sloan Digital Sky Survey uses the five filters that define the $u^{\prime} g^{\prime} r^{\prime} i^{\prime} z^{\prime}$ system. These filters have central wavelengths equal to, respectively, $0.354,0.477,0.6230,0.7630$ and $0.913 \mu \mathrm{m}$. This filter set was designed for minimum superposition and together cover a spectral interval from 0.3 to $1.123 \mu \mathrm{m}$ (Fukugita et al. 1996), which is compatible with the spectral interval used in most of the previous taxonomic classifications. To obtain information on the reflectance of the asteroids, it is necessary first to remove the solar contribution in their observed magnitudes. This is usually done by subtracting the solar colors from those obtained from the observed asteroid magnitudes, which implicitly results also on the normalization of the spectral reflectance distribution of the observation at the band used as the reference for calculating the colors. The result of this operation can be called reflectance colors:

$C_{\lambda_{j}}=-2.5\left(\log _{10} R_{\lambda_{j}}-\log _{10} R_{\lambda_{\mathrm{ref}}}\right)$,

where $C_{\lambda_{j}}$ and $R_{\lambda_{j}}$ are the reflectance color and the reflectance at a given filter, and $R_{\lambda_{\text {ref }}}$ is the reflectance at the reference filter, respectively.

Normalization was also a necessary step for reflectance measurements obtained through visible spectroscopy, since i) the spectra were not usually flux-calibrated and ii) the observed fluxes depend both on the reflectance of the surface (as a function of wavelength) and on the total illuminated area of the asteroid that was visible at the instant of the observation, which is usually unknown. The latter reason still applies to the flux-calibrated SDSS magnitudes.

After removal of the solar contribution, these reflectance colors can be used directly to forge a taxonomic scheme (Binzel et al. 2007) or can first be converted to reflectance spectra (Roig \& Gil-Hutton 2006; Misra \& Bus 2008), which is more directly comparable to what is obtained through low-resolution spectroscopy. In either case, each observation ends up parameterized by four quantities (since the reflectance at the reference filter becomes redundant) whose values depend on the choice of the normalization point.

The choice of the normalization wavelength is usually done so as to avoid regions with spectral features, in order to minimize the effects of normalization when comparing spectra. A common choice for low-resolution spectra is to normalize it at the central wavelength of the Johnson $V$ band $(0.55 \mu \mathrm{m})$, while most authors working with SDSS asteroid observations choose to normalize at the $r^{\prime}$ band (whose central wavelength is $0.6230 \mu \mathrm{m}$ ). It has been recently realized, however, (Carvano et al., in preparation) that normalization can affect spectral comparison even if made at these relatively featureless spectral regions. To avoid this potential pitfall we parameterize each observation by its reflectance color gradients, which we define as

$\gamma_{j}=-0.4 \frac{C_{\lambda_{j+1}}-C_{\lambda_{j}}}{\lambda_{j+1}-\lambda_{j}}$

These four quantities, which are independent of the normalization wavelength, represent the spectral inclination of the logarithm of the reflectance between contiguous filters. Therefore, a positive value indicates an increase in the reflectance, a negative indicates decrease in the reflectance and zero indicates that the reflectance do not varies between the filters in consideration. The reflectance colors for the $u^{\prime}$ filter can be readily calculated from the SDSS magnitudes as

$C_{u}=m_{u}-m_{g}-C_{\odot_{u g}}$

where $C_{\odot_{u g}}$ are solar colors from Ivezić et al. (2001) and the $g^{\prime}$ filter is used as reference. The color for the other filters are calculated in the same fashion and the uncertainties for $C$ and $\gamma$ are obtained by direct propagation of the uncertainty for the magnitudes.

\subsection{Classification algorithm}

Once the data parameterization is defined there are several possible paths to a supervised classification scheme. Binzel et al. (2007) use previously classified asteroids to define templates for each class and then classify the remaining observations based on the distance to the templates of each class, calculated with 
a given metric; Roig \& Gil-Hutton (2006) perform a principal component analysis on the SDSS reflectance spectra and then use previously classified asteroids to define the locus of each class in principal component space. Misra \& Bus (2008) use previously classified asteroids and SDSS observations classified by an human operator to train a neural network that, in turn, classify the remaining SDSS observations. We believe, however, that the existing schemes do not contemplate an important characteristics of the SDSS asteroid observations: that the classification can be very sensitive to magnitude uncertainties when compared to classifications made from spectroscopy.

This issue arises from the low spectral resolution of the SDSS observations. Although each observation is fluxcalibrated, hence better corrected for atmospheric extinction and systematic effects than most asteroid spectra obtained in surveys, the fact that each reflectance spectra is sampled at only 5 wavelengths means that the uncertainties in the measurements can substantially change the shape of the resulting spectra. This induces a fuzziness into the classification procedure that, we believe, must be made explicit by the classification algorithm to allow informed decisions by anyone that uses the taxonomy for target selection and/or statistical studies.

To satisfy this requirement we devised the following scheme: initially, limits on the values of the four reflectance color gradients are calculated for each class, using observations of previously classified asteroids, meteorite spectra, or synthetic spectra (see Sect. 2.3). To classify one SDSS observation of an asteroid, we calculated the probability distribution of each reflectance color gradient as a normal distribution with mean equal to $\gamma$ and standard deviation equal to the uncertainty of the measurement. The probability that the measurement in a given spectral interval is within the limits of a class is then the area of the density distribution that falls within those limits. The probability that an observation is compatible with a class is then the product of the probabilities at each spectral range. Because of the overlapping of the limits of the classes, the probabilities of the observation being compatible with different classes will not in general sum up to unity. The best classification for the observation is chosen as the one with at least $60 \%$ of the sum of the probabilities for all possible classes. The final classification is then presented as a letter for the class plus the corresponding probability. If no class satisfy this requirement (meaning that the probabilities for the observation to be compatible with two or more classes are comparable), all classes with probabilities over $30 \%$ of the total probability are considered possible and a multiple letter classification is issued, with the corresponding probability being the average value for the possible classes. The order of the letters in multiple classification has no relation with the probability of each class. If an observation fails to be assigned to any class, it is labeled as unclassified ("U").

One consequence of this classification scheme is that observations with high observational uncertainties in one or more filters will be classified with low probabilities. These classifications will then be easily recognized as problematic, but could still be useful in some contexts.

Other distinctive characteristic of the SDSS MOC4 is that it includes several asteroids with multiple observations, which increases the possibility of having asteroids with multiple classifications. Since a unique classification for each asteroid is needed for statistical studies, whenever there is variation in the taxonomic class with different observations, we adopt for the asteroid the most frequent taxonomy, with probability equal to the higher value for that class. If two classes are equally frequent, a multiple letter classification is issued, with probability equal to the mean of the higher probabilities for each class, and a flag is set to indicate that this situation has happened.

\subsection{Definition of the classes}

A final step is to choose which classes of the spectroscopic taxonomies to represent and how to define their limits. Another problem here is the nomenclature: the norm in the literature is for new taxonomic schemes to use the same designations for their classes that were used in previous taxonomies, even though they usually differ in the way they are defined. Here we append a " $p$ " to the corresponding designation in the Bus taxonomy, to indicate clearly that this taxonomy is obtained from photometric data. We decided to define 9 classes, based on their current mineralogical interpretation and the ability of the SDSS data to represent them. The adopted classes are $V_{\mathrm{p}}, O_{\mathrm{p}}, Q_{\mathrm{p}}, S_{\mathrm{p}}, A_{\mathrm{p}}, \mathcal{L}_{\mathrm{p}}$, $D_{\mathrm{p}}, X_{\mathrm{p}}$, and $C_{\mathrm{p}}$. Except for the $\mathcal{L}_{\mathrm{p}}$ class, all others are intended to be directly comparable to the corresponding classes in Bus taxonomy. It should be noted that, although this classification scheme was designed to be compatible with the Bus taxonomy, such compatibility is limited by the characteristics of the dataset.

The first five are characterized by an absorption band around $1 \mu \mathrm{m}$, associated with olivine/pyroxene assemblages. On the SDSS data, this band causes as a drop in reflectance at the $z^{\prime}$ filter. The $V$ class characteristic spectrum is distinctive by having the deepest $1 \mu \mathrm{m}$ band among observed asteroid spectra. This class is firmly associated with HED meteorites (McCord et al. 1970), and is observed mostly in and around the Vesta family (Binzel \& Xu 1993; Burbine et al. 2001; Florczak et al. 2002; Alvarez-Candal et al. 2006) in the inner main belt, with a few observed in the intermediate (Binzel et al. 2006; Roig et al. 2008; Moskovitz et al. 2008; Nesvorný et al. 2008) and outer belt (Lazzaro et al. 2000; Duffard \& Roig 2009). The O and $\mathrm{Q}$ classes are characterized by a not very deep $1 \mu \mathrm{m}$ band and by a flatter spectrum shortwards the $i^{\prime}$ band than the $\mathrm{V}$ class (with the $\mathrm{Q}$ class being more reddened in the region than the $\mathrm{O}$ class). Both have been associated with ordinary chondrite meteorites (McFadden et al. 1984, 1985; Bell \& Keil 1988; Binzel et al. 1993), since their visible spectra is similar to spectra of this type of meteorite (Burbine \& Binzel 2002).

Direct comparison and mineralogical inferences based on NIR spectra tend to confirm this association for the Q class (Binzel et al. 2001), but the situation is not as clear for the O class (Chapman 1996a; Binzel et al. 2001; Burbine \& Binzel 2002; DeMeo et al. 2009). Both classes are rare among asteroids, with Q-class asteroids observed mostly among the NEO population (Binzel et al. 2004; Mothé-Diniz \& Nesvorný 2008). The $\mathrm{S}$ class, on the other hand, is the most common for asteroids in the inner and intermediate main belt (Mothé-Diniz et al. 2003). This class harbors a good deal of spectral diversity, and in the Bus taxonomy (Bus \& Binzel 2002a) it was deemed a "complex" composed of 6 subtypes. Spectra of asteroids with this classification tend to be redder than the Q-class shortward of the $i^{\prime}$ band, present a shallower $1 \mu \mathrm{m}$ band, and, in general, are not well-matched by most meteorite spectra. Mineralogical inferences made based on the position and area of the 1 and $2 \mu \mathrm{m}$ olivine/pyroxene and pyroxene bands suggest that a considerable mineralogical diversity can be found among the asteroids with this classification, including ordinary chondrites, mixtures of metal and basalts and olivine-dominated assemblages (Gaffey et al. 1993). Further evidence from laboratory experiments (Hapke 2001; Sasaki et al. 2001) and spacecraft observations (McFadden et al. 1985) suggest that at least part of the S-class asteroids may have a superficial composition compatible 
with ordinary chondrite material affected by space weathering processes.

Finally, A-class asteroids present extremely reddened spectra shortwards the $i^{\prime}$ band and a moderate to deep $1 \mu \mathrm{m}$ band. These asteroids are rare in the main belt, and their characteristic spectrum is not well-matched to spectra of meteorites (Burbine 2000). Analysis from NIR spectra shows that A-class asteroids have compositions dominated by olivine, with varying fosterite content. These extremes may represent mantle material from a completely differentiated parent body or material similar in composition to R chondrites (Sunshine et al. 2007).

The $\mathrm{D}, \mathrm{X}$, and $\mathrm{C}$ classes show no clear $1 \mu \mathrm{m}$ band, and their spectra longwards of the $g^{\prime}$ band is mostly featureless. Their main defining characteristic is their spectral gradient in this region, with the $\mathrm{D}$ class presenting a very steep gradient, the $\mathrm{X}$ class a moderate gradient, and the $\mathrm{C}$ class a flat to slightly bluish gradient (Bus \& Binzel 2002a). Given the absence of mineralogically diagnostic bands in most of the cases, mineralogical inferences for these classes rely mostly on comparison with meteorite spectra and synthetic spectra generated from mixing models. The D-type asteroids are generally believed to be mixtures of ices, anhydrous silicates, and organics. Their characteristic spectra is mostly not seem among meteorites with the exception of a slight similarity with some iron meteorites (Carvano et al. 2003) and the Tagish Lake meteroite (Hiroi et al. 2003), which is an ungrouped carbonaceous chondrite. Also, the D-class are the predominant class in the far end of the outer main belt and in the Trojan region, and their spectra are similar to spectra of part of the TNOs and comet nuclei (Burbine 2000). The X and C classes, on the other hand, show some spectral diversity and were also deemed "complexes" in the Bus taxonomy, each subdivided into several subclasses. Comparison to meteorites (Burbine 2000) suggest that their possible mineralogies include all carbonaceous chondrites subtypes, shock-darkened ordinary chondrites, enstatite chondrites, iron meteorites, and some achondrites. Also, spectra similar to both classes can be seen among TNOs and comet nuclei (Jewitt 2002), so that volatile and organic rich compositions are also a possibility. Notable exceptions to this general indetermination is the $\mathrm{Ch}$ Bus subclass, characterized by a wide absorption band around $0.7 \mu \mathrm{m}$ that is associated to water alteration in mafic phyllosilicates and that is present on some CM carbonaceous chondrites (Vilas \& Gaffey 1989).

Finally, the $\mathcal{L}_{\mathrm{p}}$ class, as defined in this work, differs from the other because it is not directly comparable to classes defined in previous works. Rather, it is defined by the junction of the $\mathrm{K}$, $\mathrm{L}$, and Ld classes from Bus taxonomy, and for this reason, it is represented here by a different typesetting. These three classes have spectra that, shortwards of the $i^{\prime}$ band, vary from moderately $(\mathrm{K})$ to extremely reddened (Ld), all presenting a flattening of the spectra longward of that band. This last characteristic may stem from the presence of a very shallow $1 \mu \mathrm{m}$ band, and in this sense it represents a transition between the classes with and without the band.

\subsection{Defining the limits of the classes}

A first step is to select the objects that have spectroscopic classification in the Bus system and that have also been observed by the SDSS. We use here the objects observed by the SMASSII (Bus \& Binzel 2002b) and S3OS2 surveys (Lazzaro et al. 2004). Since we want these objects to define each class, it is important to exclude object whose classification is known to change between observations. The issue of spectral variations among the
Table 1. Number of objects with unambiguous spectral classification that were observed by the SDSS.

\begin{tabular}{cccc}
\hline \hline Class & Unambiguous Class. & Asteroids in SDSS & SDSS Observ. \\
\hline $\mathrm{V}$ & 42 & $11(11)$ & $35(32)$ \\
$\mathrm{A}$ & 17 & - & - \\
$\mathrm{S}$ & 741 & $153(128)$ & $304(237)$ \\
$\mathrm{Q}$ & 7 & - & - \\
$\mathrm{O}$ & 3 & - & - \\
$\mathcal{L}$ & 117 & $33(27)$ & $79(66)$ \\
$\mathrm{C}$ & 451 & $93(86)$ & $191(167)$ \\
$\mathrm{X}$ & 383 & $97(91)$ & $176(164)$ \\
$\mathrm{D}$ & 99 & $27(25)$ & $50(42)$ \\
\hline
\end{tabular}

two spectroscopic surveys is discussed in Carvano et al. (2003) and in Lazzaro et al. (2004). Using the compilation of taxonomic classification of asteroids (Neese 2006), we looked for objects that have classifications in the classes that form the classes we adopt here. If an object was observed in both spectroscopic surveys, it was only used if its classifications according to both fall in the same class. We then examined the distribution of the uncertainties in the color gradients of the whole sample: observations where the errors were greater than the 3rd quartile for any of the filters were discarded at this stage. The result of this search is shown in Table 1. In this table, the third and fourth columns the number in parenthesis refer to the number of objects and their observations that remained after removing the observations with uncertainties above the adopted upper limit.

We note that no A-, Q-, and O-type asteroids with unambiguous taxonomy have been observed by the SDSS. Q- and Otypes are thought to represent the unweathered end-members of the population of asteroids with ordinary chondrite composition. It is then reasonable to use meteorite spectra to obtain representative SDSS reflectances for these classes. To do so, we used the templates of the Q and O classes in Bus taxonomy to search the RELAB database for OC meteorites with a spectrum similar to each class, with the methodology described in Mothé-Diniz \& Carvano (2005). SDSS reflectances for each meteorite were then obtained by convolution of the meteorite spectra with the SDSS band passes for each filter. This approach is not possible for A-type asteroids, since they have no clear analogs among the meteorite collection. However, it is well established that A-type asteroids are olivine-dominated assemblages (Sunshine et al. 2007), with compositions ranging from magnesium-rich to iron-rich. Therefore, to obtain reflectances in the SDSS range we adopted the following approach: for each of the 16 unambiguous A-type visible spectra, we used the implementation of the Modified Gaussian Model (Sunshine et al. 1990) described in Mothé-Diniz et al. (2008) to fit olivine bands. Since the visible spectrum alone is not sufficient to allow a unique fit, the three olivine bands were fixed to a given fosterite composition by both the band center calibration and the band-depth ratio calibration (Sunshine et al. 2007). For each spectrum, we then produced five synthetic spectra by fitting with compositions $F O_{10}, F O_{20}$, $\mathrm{FO}_{50}, \mathrm{FO}_{70}$, and $\mathrm{FO}_{90}$. These synthetic spectra were then convolved with the SDSS band passes to yield the SDSS reflectance spectra for the A-class.

To check whether the reflectance gradient ranges of the template spectra of each class are able to be used as classification criteria we inspected for superpositions. We did that by calculating the kernel density plot for each class at each spectral range. This is done in the following fashion: the density of probability of a given observation of an object of a given class having a reflectance gradient at a given value was calculated as a normal 
distribution, with the mean equal to the reflectance gradient of the observation and the variance equal to the uncertainty in the reflectance gradient. The density distribution of the class in that range was then given by the sum of the densities of all observations of asteroids of that class. To use the proposed scheme for classification, it was necessary that each class presents little superposition with all the other in at least one spectral interval. However, since the classes represent a continuous distribution, some degree superposition is unavoidable in some instances.

It is found that there is considerable overlapping between the $S_{\mathrm{p}}, \mathcal{L}_{\mathrm{p}}$ and $D_{\mathrm{p}}$ classes, in particular between the $S_{\mathrm{p}}$ and the $\mathcal{L}_{\mathrm{p}}$ classes and between the $\mathcal{L}_{\mathrm{p}}$ and the $D_{\mathrm{p}}$ classes. Upon visual inspection of the spectra, we see that several asteroids classified as S-type present in some observations SDSS reflectance spectra, which are closer to templates of the $\mathrm{L}$ and $\mathrm{K}$ classes, with a flattening of the reflectance towards $1 \mu \mathrm{m}$. Conversely, many SDSS observations of asteroids in the $\mathcal{L}_{\mathrm{p}}$ class show spectra more similar to S-type asteroids, with a decrease in the reflectance towards $1 \mu \mathrm{m}$. Similarly, the overlapping between the $D_{\mathrm{p}}$ and $\mathcal{L}_{\mathrm{p}}$ classes happens mostly because of the Ld-class asteroids in the latter. Both Ld- and D-type spectra have steep visible spectral gradients, but for the Ld-class there is a flattening of the reflectance towards $1 \mu \mathrm{m}$, whereas the reflectance of the D-type asteroids shows a continued increase in that range.

A solution is to seting strict limits for reflectance gradients of the $S_{\mathrm{p}}, \mathcal{L}_{\mathrm{p}}$, and $D_{\mathrm{p}}$ classes templates in the $(z-i)$ range. A natural choice of theses limits are the values of the reflectance gradient where the density distribution of the classes intercept each other. Observations of asteroids classified as the $S_{\mathrm{p}}, \mathcal{L}_{\mathrm{p}}$ and $D_{\mathrm{p}}$ classes by spectroscopic surveys that fall outside these limits are not used to define the classes from SDSS reflectance color gradients.

The resulting densities are then used to define the final limits of the classes. The limits of each class are, at this step, given by the region in each spectral range that contains a $90 \%$ probability of containing a template of that class. Figure 1 shows schematically the limits of the classes in reflectance gradient space. The log reflectance spectra corresponding to the peak of the distributions for each class is shown in Fig. 2.

\section{Results}

\subsection{Comparison with spectroscopic classifications}

It is instructive to apply this classification scheme first to the sample of asteroids with unambiguous classification. Here we use the whole sample, with no exclusions based on the uncertainties. Figure 3 shows the distribution of the classification made from the SDSS observations of the objects with spectroscopic classification for each class.

In this figure, observations for which the higher probability was lower than $5 \%$ were considered to be unclassified (U). Also, the amount of observations in each class made from observations considered bad are shown separately.

In general, visual inspection of the spectra shows that they are consistent with the assigned class. Exceptions are the spectroscopic $\mathrm{C}$ asteroids that have observations classified as $D_{\mathrm{p}}$ or $C D_{\mathrm{p}}$, which would fit in the $C_{\mathrm{p}}$ or $X_{\mathrm{p}}$ classes better. Most of the observations that failed to be classified have high uncertainties. Visual inspection of the observations assigned to the $U$ class that have uncertainties below the threshold adopted show that all are definitively "strange".
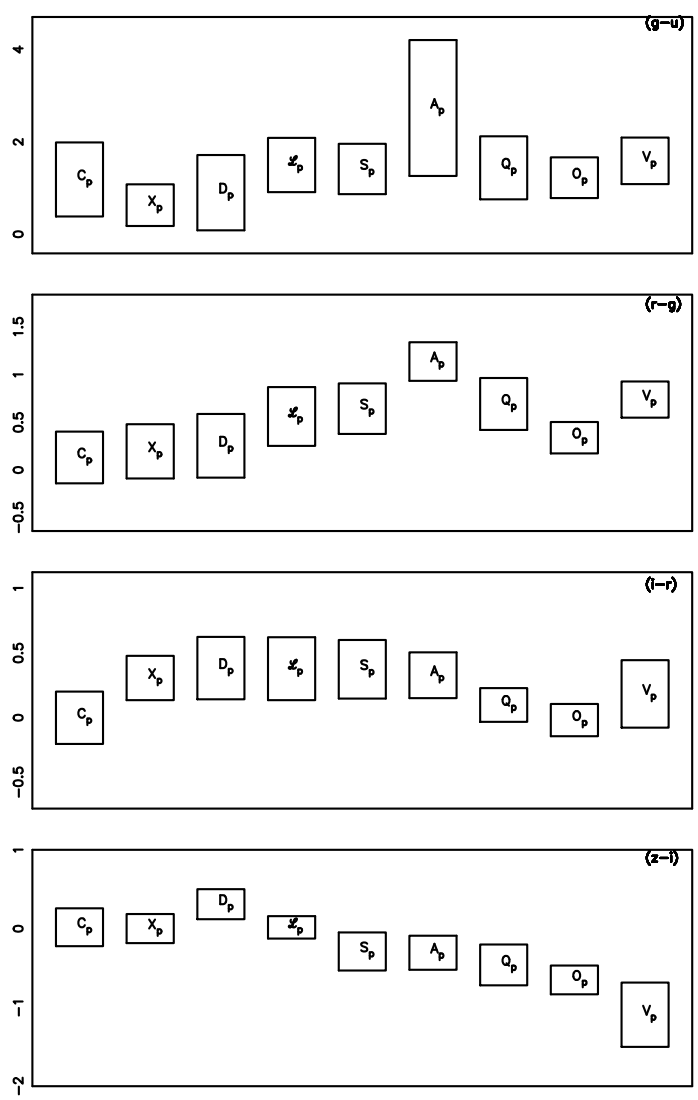

Fig. 1. Final reflectance color gradient limits for the classes.

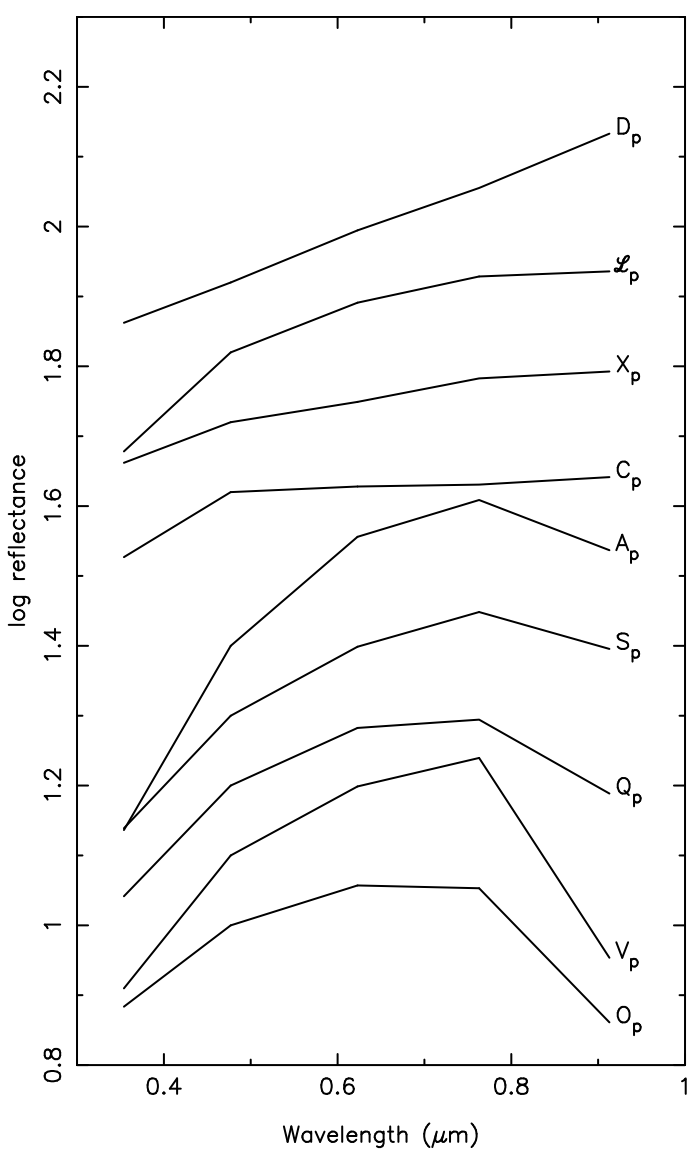

Fig. 2. Median log reflectance spectra for the classes, shifted vertically for clarity. 

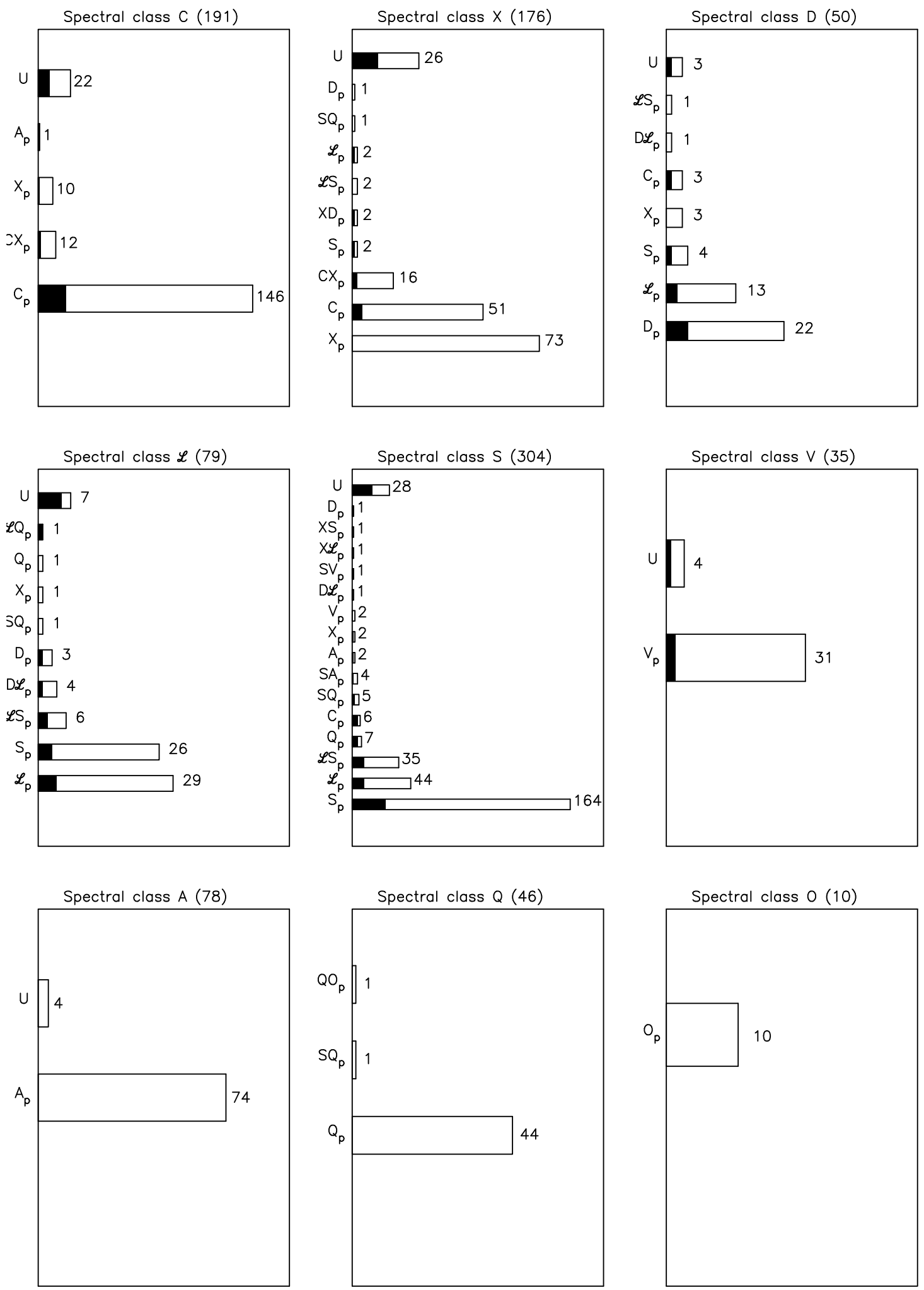

Fig. 3. Distribution of the SDSS taxonomic classes for observations of the asteroids previously classified based on spectroscopy. The solid filling represents the number of observations with uncertainties above the threshold set in Sect. 2.4.

\subsection{Application to meteorite spectra}

Direct comparison between meteorite and asteroid spectra is hampered by the effects of space weathering processes acting on the surface of the asteroids. This effect, together with the selectiveness of the transport mechanisms of meteoroid are currently understood as the main culprits for the observed mismatch between the spectral properties of asteroids and meteorites (Gaffey et al. 2002). Even so, such an exercise can provide some insight to the mineralogical interpretation of the taxonomic classes. To this end, we calculated the SDSS color (see Sect. 2.4) and applied the classification algorithm derived in the previous sessions 
J. M. Carvano et al.: SDSS-based taxonomic classification
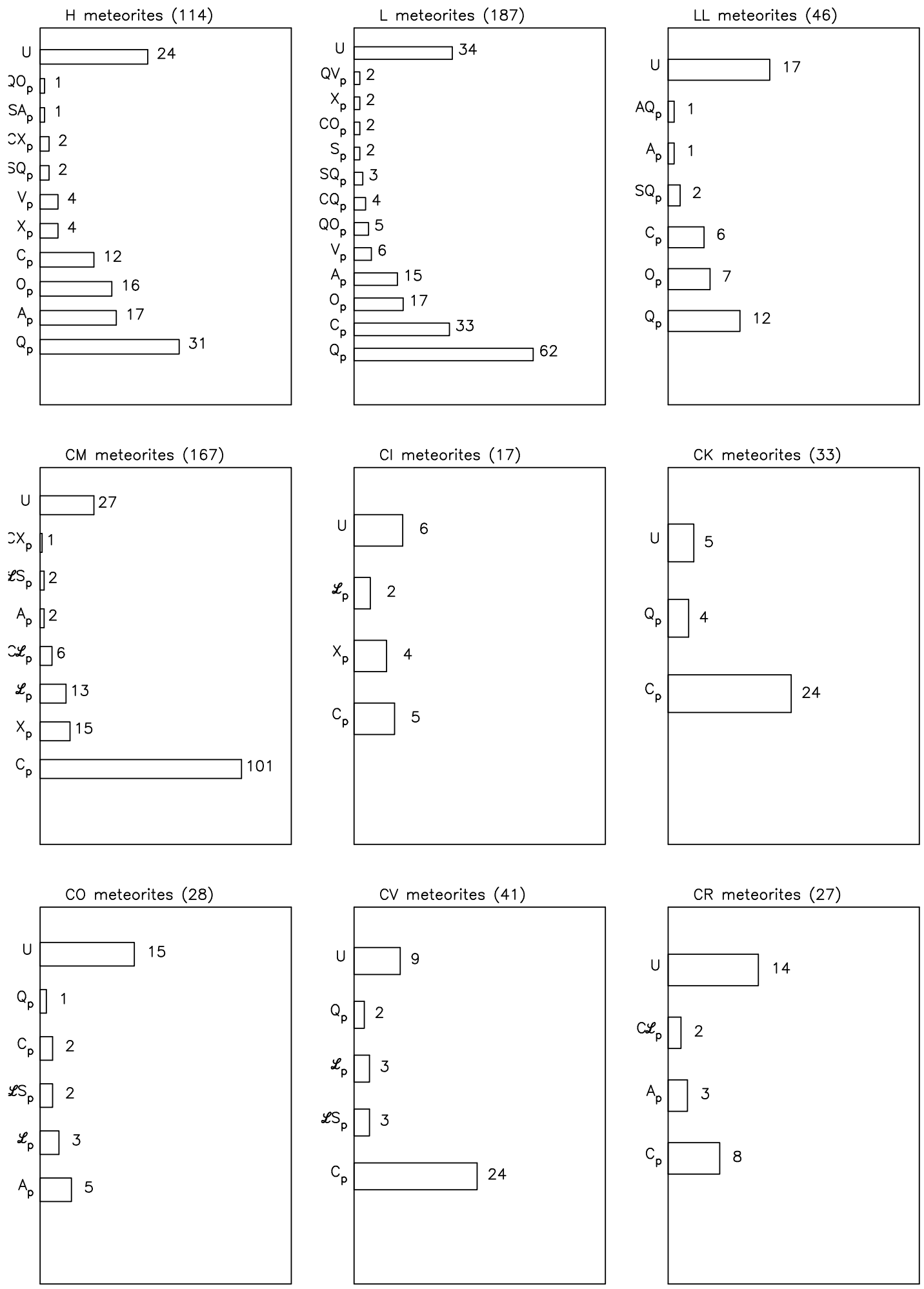

Fig. 4. Distribution of the SDSS taxonomic classes for meteorite spectra from the RELAB database.

to a sample of bidirectional reflectance spectra of several meteorite classes from the RELAB spectroscopic database (Pieters \& Hiroi 2004). For each spectral interval, we used the median uncertainties from the sample of asteroids with previous spectroscopic classification that were observed by the SDSS. This essentially tells us what would be the taxonomic classification of asteroids covered by unweathered material of each meteorite type.
The results are shown as histograms in Figs. 4 and 5. From these figures, it is clear that none of the meteorite types is spectroscopically homogeneous, with specimens falling into different SDSS taxonomic classes. There is also many of spectra that fail to be classified. Visual inspection reveals that the main culprit for most of the unclassified meteorite spectra is the steep decrease in the reflectance towards the UV that is commonly seen in meteorite spectra but rare among asteroids (Britt et al. 1992; 

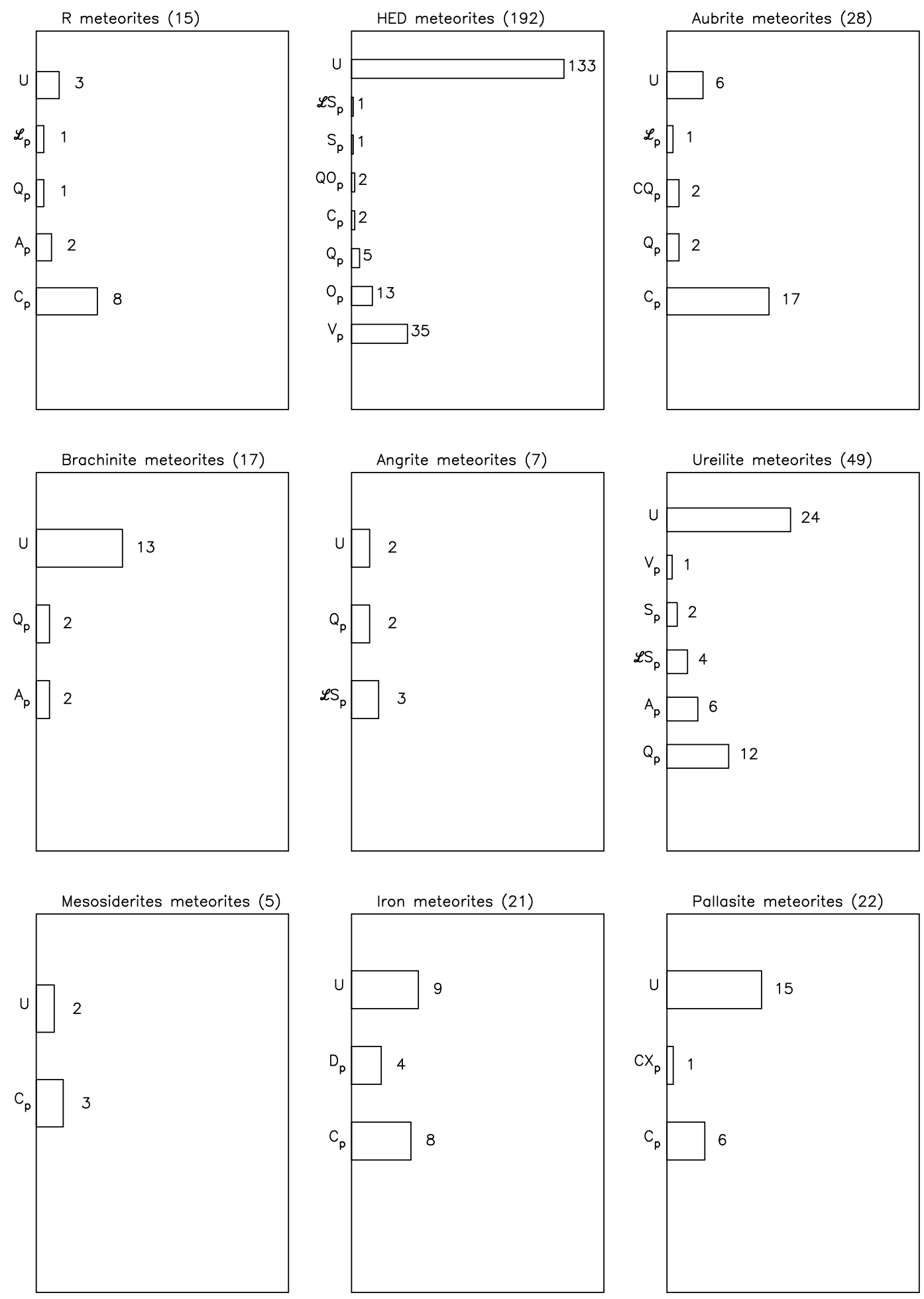

Fig. 5. Distribution of the SDSS taxonomic classes for meteorite spectra from the RELAB database.

Burbine 2000; Carvano et al. 2003). This characteristic is also responsible for the classification of some carbonaceous chondrite and ordinary chondrite spectra in the $A_{\mathrm{p}}$ class.

As expected, most ordinary chondrites fall into the $Q_{\mathrm{p}}$ or $O_{\mathrm{p}}$ classes, but not exclusively: the spectra of some $\mathrm{H}$ and $\mathrm{L}$ chondrites with unusually deep $1 \mu \mathrm{m}$ band end up classified as $V_{\mathrm{p}}$-class. Also, a number of spectra with unusually swallow $1 \mu \mathrm{m}$ band are assigned to the $C_{\mathrm{p}}$ or $X_{\mathrm{p}}$ classes. These are mostly spectra of shock-darkened ordinary chondrites, also known as black chondrites (Britt \& Pieters 1989). Also as expected, the majority of HED spectra that end up classified were assigned to the $V_{\mathrm{p}}$ class, but a few spectra with shallower $1 \mu \mathrm{m}$ fell into the $O_{\mathrm{p}}$ and $Q_{\mathrm{p}}$ classes. Besides the UV drop in reflectance, the large number of HED spectra that could not be classified is also a consequence of a $1 \mu \mathrm{m}$ that is deeper than what is seen among V-class asteroid spectra in Bus taxonomy. Most spectra of carbonaceous chondrites subtypes fall into the $C_{\mathrm{p}}$ class, although a notable exception are the $\mathrm{CO}$ carbonaceous chondrites that show more affinity for the $A_{\mathrm{p}}, \mathcal{L}_{\mathrm{p}}$ and $\mathcal{L} S_{\mathrm{p}}$ classes. Also predominantely classified as $C_{\mathrm{p}}$-class are the spectra of mesosiderites, 
Table 2. Number of asteroids and observations classified.

\begin{tabular}{crr}
\hline \hline Classification & Csteroids & Observations \\
\hline$C_{\mathrm{p}}$ & $17764(3357)$ & $28784(5328)$ \\
$X_{\mathrm{p}}$ & $3558(1133)$ & $6003(1787)$ \\
$D_{\mathrm{p}}$ & $2580(377)$ & $4268(656)$ \\
$\mathcal{L}_{\mathrm{p}}$ & $4815(976)$ & $8129(1603)$ \\
$S_{\mathrm{p}}$ & $15581(3784)$ & $25713(5867)$ \\
$A_{\mathrm{p}}$ & $410(33)$ & $883(87)$ \\
$V_{\mathrm{p}}$ & $2818(561)$ & $4823(917)$ \\
$Q_{\mathrm{p}}$ & $3296(325)$ & $5669(647)$ \\
$O_{\mathrm{p}}$ & $63(15)$ & $155(29)$ \\
$C X_{\mathrm{p}}$ & $2284(432)$ & $4082(819)$ \\
$X D_{\mathrm{p}}$ & $484(51)$ & $856(108)$ \\
$D \mathcal{L}_{\mathrm{p}}$ & $615(22)$ & $1045(49)$ \\
$\mathcal{L} S_{\mathrm{p}}$ & $5151(782)$ & $8926(1456)$ \\
$S A_{\mathrm{p}}$ & $145(14)$ & $418(28)$ \\
$S V_{\mathrm{p}}$ & $256(6)$ & $536(14)$ \\
$S Q_{\mathrm{p}}$ & $2394(295)$ & $4663(664)$ \\
$Q V_{\mathrm{p}}$ & $362(19)$ & $716(43)$ \\
\hline
\end{tabular}

pallasites and iron meteorites. The $D_{\mathrm{p}}$-class spectra are seen among $\mathrm{CI}$ and $\mathrm{CM}$ and (just one case) L ordinary chondrite and also among iron meteorites. It is interesting to note that, apart from 2 L chondrites, 2 urellites and 1 HED spectra, spectra compatible with the $S_{\mathrm{p}}$ class, are not seen among meteorites, which agrees with the current view that the S-type spectra are a consequence of space weathering processes acting on ordinary chondrite (Chapman 1996b) and possibly other mineralogies.

\subsection{Application to the MOC4}

We proceed then to apply the classification scheme to all observations of asteroids in the MOC4. Here we do not exclude any observation a priori, but only flag as bad the ones with uncertainties above the threshold. The MOC4 contains 218996 observations of 104687 asteroids. Out of these, a total of $107466 \mathrm{ob}-$ servations of 63468 asteroids are classified in this fashion. Out of the 111530 observations that failed to be classified, 109559 had one or more filters with uncertainties above the threshold, while 87002 observations flagged as bad were still classified with probability greater than $5 \%$. Table 2 shows the number of asteroids and observations classified into each of the adopted classes and also into some of the most common ambiguous classifications. As in Table 1, the number inside parenthesis refer to the asteroids and observations with uncertainties below the threshold - for asteroids this is the number of objects whose best classified observation have uncertaities above the threshold.

\subsubsection{Taxonomic variations}

Color variation among observations of asteroids in the SDSS have been analyzed by (Szabó et al. 2004) for a sample of 7531 asteroids in the third release of the MOC. The authors conclude that the observed variations are real and caused by surface inhomogeneities in the asteroids, rather than by observational artifacts or random errors. Here we use a much bigger sample to analyze color variations that lead to different taxonomic classifications between observations.

A total of 22019 asteroids have two or more observations with successful classifications. Of these, 14962 show taxonomic variations. Table 3 shows the number of asteroids associated with each combination of taxonomies found, for the combinations that are seen on 100 asteroids or more. As should be
Table 3. Objects with taxonomic variation between observations.

\begin{tabular}{cc}
\hline \hline Classes observed & Number of asteroids \\
\hline $\mathcal{L} S_{\mathrm{p}}$ & 3057 \\
$C X_{\mathrm{p}}$ & 2325 \\
$S Q_{\mathrm{p}}$ & 1515 \\
$\mathcal{L} S Q_{\mathrm{p}}$ & 952 \\
$D \mathcal{L} S_{\mathrm{p}}$ & 386 \\
$Q V_{\mathrm{p}}$ & 334 \\
$S Q V_{\mathrm{p}}$ & 328 \\
$C X D_{\mathrm{p}}$ & 303 \\
$S V_{\mathrm{p}}$ & 278 \\
$D \mathcal{L}_{\mathrm{p}}$ & 250 \\
$X D_{\mathrm{p}}$ & 243 \\
$X \mathcal{L} S_{\mathrm{p}}$ & 219 \\
$\mathcal{L} A S_{\mathrm{p}}$ & 193 \\
$A S_{\mathrm{p}}$ & 186 \\
$C D_{\mathrm{p}}$ & 159 \\
$X S_{\mathrm{p}}$ & 153 \\
$C S_{\mathrm{p}}$ & 152 \\
$D S_{\mathrm{p}}$ & 141 \\
$X \mathcal{L}_{\mathrm{p}}$ & 139 \\
$C S Q_{\mathrm{p}}$ & 129 \\
$C Q_{\mathrm{p}}$ & 123 \\
$C X \mathcal{L}_{\mathrm{p}}$ & 120 \\
$C \mathcal{L} S_{\mathrm{p}}$ & 109 \\
$\mathcal{L} A S Q_{\mathrm{p}}$ & 100 \\
\hline
\end{tabular}

expected, most of the variations seen are between classes with adjacent limits in one or more spectral intervals. This is the case of the most frequent transitions, from $\mathcal{L}_{\mathrm{p}}$ to $S_{\mathrm{p}}$, from $C_{\mathrm{p}}$ to $X_{\mathrm{p}}$ and from $S_{\mathrm{p}}$ to $Q_{\mathrm{p}}$, and the transitions from $D_{\mathrm{p}}$ to $\mathcal{L}_{\mathrm{p}}$ and from $X_{\mathrm{p}}$ to $D_{\mathrm{p}}$, from $Q_{\mathrm{p}}$ to $V_{\mathrm{p}}$ and $S_{\mathrm{p}}$ to $V_{\mathrm{p}}$ also fall into this category. Such variations can be caused by random errors for the objects with spectra falling close to the classes limits, but they can also reflect real surface variations, caused either by variations in the prevailing mineralogy or variations in the prevailing degree of space weathering maturity. Alternatively, some of the variations can also be caused by differences in the solar phase angle among observations and phase-reddening effects.

More interesting are the transitions between more dissimilar classes, $D_{\mathrm{p}}$ to $S_{\mathrm{p}}, A_{\mathrm{p}}$ to $S_{\mathrm{p}}, X_{\mathrm{p}}$ to $S_{\mathrm{p}}, C_{\mathrm{p}}$ to $S_{\mathrm{p}}$ and $C_{\mathrm{p}}$ to $Q_{\mathrm{p}}$. These transitions imply that those asteroids at some time presented spectra dominated by olivine/pyroxene bands that become suppressed at some other point of their orbit and/or rotational aspect. Possible mechanisms for accomplishing this include extreme space weathering, contamination by metal, or other spectrally dark phase, the coexistence of different mineralogies in the same body or phase reddening. Again, it is not possible to rule out random error as the cause of the taxonomic variation in some instances.

\subsubsection{Distribution of taxonomies in the main belt}

The distribution of taxonomies in the main belt can be highlighted through the analysis of the density maps for each class. To construct such maps we simply divide the main belt in bins of osculating semi-major axis and inclination and then count the number of objects of a given class inside each bin. Here we adopted bins with sides of $0.008 \mathrm{AU}$ in semi-major axis and $0.4^{\circ}$ in inclination. The usefulness of this kind of plot for analyzing the distribution of asteroids can be appreciated from Figure 6, which shows a density map made from all asteroids listed in the ASTORB file (Bowell 2009) for semi-major axes between 1.8 and 5.5 AU and inclinations up to $30^{\circ}$. The patchy nature of the 


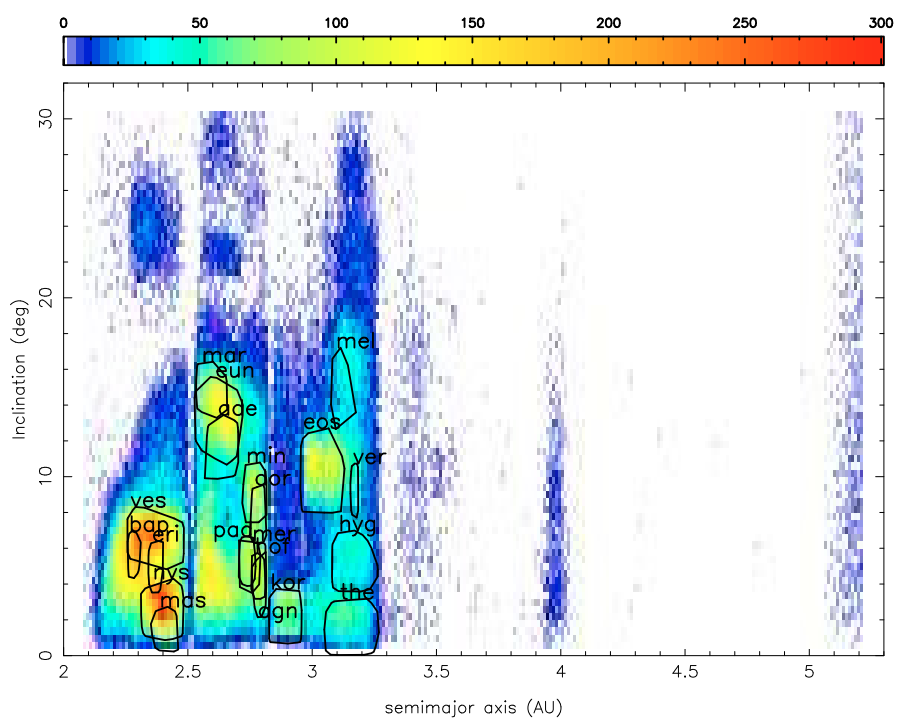

Fig. 6. Density map of all numbered asteroids. The location of several families are marked.

asteroid distribution in the main belt is clearly seen, as is the localized increase in density in the vicinity of the major asteroid families. The loci in osculating element space of the members of the families of Merxia, Veritas, Nysa, Adeona, Padua, Koronis, Maria/Renate, Mineva, Eunomia, Vesta, Erigone, Dora, Agnia, Eos, Hygiea, Baptistina, Meliboea, Hoffmeister, Massalia, and Themis (as defined in Mothé-Diniz et al. 2005) are delimited by closed lines in Fig. 6.

Figure 7 shows the density distributions for the classes $C_{\mathrm{p}}$, $X_{\mathrm{p}}, \mathcal{L}_{\mathrm{p}}, D_{\mathrm{p}}, S_{\mathrm{p}}, Q_{\mathrm{p}}, V_{\mathrm{p}}$ and $A_{\mathrm{p}}$, for all asteroids with classification probability $>5 \%$. In general, limiting the sample based on the uncertaities of the measurements produce qualitatively similar results to what is shown in Fig. 7.

This figure summarizes several results for the distribution of the taxonomies in the main belt that have been already mentioned in the literature, but also reveals some features that have not been previously discussed. The most interesting features can be summarized as follows.

- The $X_{\mathrm{p}}$ and, particularly, the $C_{\mathrm{p}}$ classes seem to dominate the background beyond 2.8 AU until the outer border of the main belt. In this region the $S_{\mathrm{p}}$-class asteroids mostly appear in the vicinities of the Koronis and Eos families. This predominance of the $C_{\mathrm{p}}$ and $X_{\mathrm{p}}$ classes in the background becomes even more explicit at inclinations greater than $20^{\circ}$.

- The $S_{\mathrm{p}}$-class asteroids, on the other hand, dominate the region before 2.8 AU, showing a considerable increase in density even in regions not associated with dynamical families. In this region the increase in the density of $C_{\mathrm{p}}$-class asteroids only occur in regions associated to dynamical families; however, unlike the $S_{\mathrm{p}}$-class in the outer belt that are rare and patchy outside families, the background $C_{\mathrm{p}}$ - and $X_{\mathrm{p}}$-class asteroids in the inner belt seem evenly distributed.

- Although their overall distribution is similar, the $X_{\mathrm{p}}$-class seems less frequently associated with large families than $C_{\mathrm{p}^{-}}$ class asteroids. The most notable exception occurs in the vicinity of the Eos family.

- $D_{\mathrm{p}}$-class asteroids dominate beyond 3.5 AU. In the Trojan region, in particular, only $D_{\mathrm{p}}$-class asteroids are observed with inclinations greater than $20^{\circ}$. In the main belt, $D_{\mathrm{p}}$-class asteroids seem evenly distributed, with two notable exceptions: they are not seen in the outer belt with inclinations greater than $20^{\circ}$, and they seem to cluster in the region of the Eos family.

- The distribution of the $Q_{\mathrm{p}^{-}}, \mathcal{L}_{\mathrm{p}^{-}}$, and $A_{\mathrm{p}}$-classes seem to follow the same pattern as the $S_{\mathrm{p}}$-type asteroids, with two noticeable exceptions: $Q_{\mathrm{p}}$-class asteroids show a clustering in the vicinity of the Vesta family that is not evident in the $S_{\mathrm{p}^{-}}$ class asteroids; the $\mathcal{L}_{\mathrm{p}}$-class asteroids show a clustering in the vicinity of the Eos family that is clearly more intense than what is seen for the $S_{\mathrm{p}}$-class asteroids and also seem to cluster in the vicinity of the Meliboea family, while no clear clustering of $S_{\mathrm{p}}$-class asteroids can be seen in this region.

- The $V_{\mathrm{p}}$-class asteroid only show a significant clustering in the vicinity of the Vesta family, but are scattered in the intermediate and outer belts.

The previous works on the distribution of taxonomies in the main belt (Gradie et al. 1989; Bus \& Binzel 2002a; Mothé-Diniz et al. 2003) have focused mostly on the heliocentric distribution of the classes, all showing a predominance of S-type asteroids in the inner belt, an increase in the fraction of $\mathrm{C}$ as the heliocentric distance increases, and the dominance of D-type spectra beyond 3.5 AU. Mothé-Diniz et al. (2003) were the first to discuss how such distribution is affected by the orbital eccentricity and inclination of the objects showing less spread of the class distribution at higher inclinations and eccentricities than at lower ones. In the present work, using a larger sample of objects and two-dimensional density maps potentially allow a more detailed insight into the distribution of taxonomies. One interesting feature that can be seen in Fig. 7 is the fact that only $C_{\mathrm{p}}$ and $X_{\mathrm{p}}$ asteroids are seen at high inclinations between 3 and 3.5 AU. Such trends in the distribution of background objects may possibly provide additional constraints to models of the early evolution of the main belt. A major hurdle here is the pollution of the background distribution by the presence of asteroid families. The possibility of existence of taxonomic diversity inside asteroid families is also interesting since it may be the signature for the breakup of differentiated bodies. The reality of such differentiated families is controversial. Although models of the early evolution of the main belt suggest the presence of large planetary embryos during the early ages (O'Brien et al. 2007) that may have suffered catastrophic disruptions, most of the asteroid families seem to be fairly homogeneous (Bus 1999; Cellino et al. 2002; Mothé-Diniz et al. 2005), with the most notable exceptions being the Eos and Maria Family (Mothé-Diniz et al. 2005). Recent works that use SDSS colors to study families (Parker et al. 2008) also tend to report color homogeneity in the families, although it should be noted that they use the SDSS colors as a criterion to define the family membership. It is possible that the taxonomic diversity in the Eos and in other families that are seen in Fig. 7 may stem from the overlapping of debris from several breakup events. Further dynamical studies made without the previous assumptions about spectral homogeneity are required to settle this question. Such studies may benefit from the dataset presented here.

\section{Conclusions}

Here we have developed a taxonomic scheme that is compatible with the Bus taxonomy and that is suitable to the peculiarities of the SDSS observations. In particular, it explicitly takes into account the uncertainty in the classification introduced by the low spectral resolution of the SDSS data by assigning probabilities to each classification. In this way, it is possible to maximize the number of classified objects while allowing the putative users to 

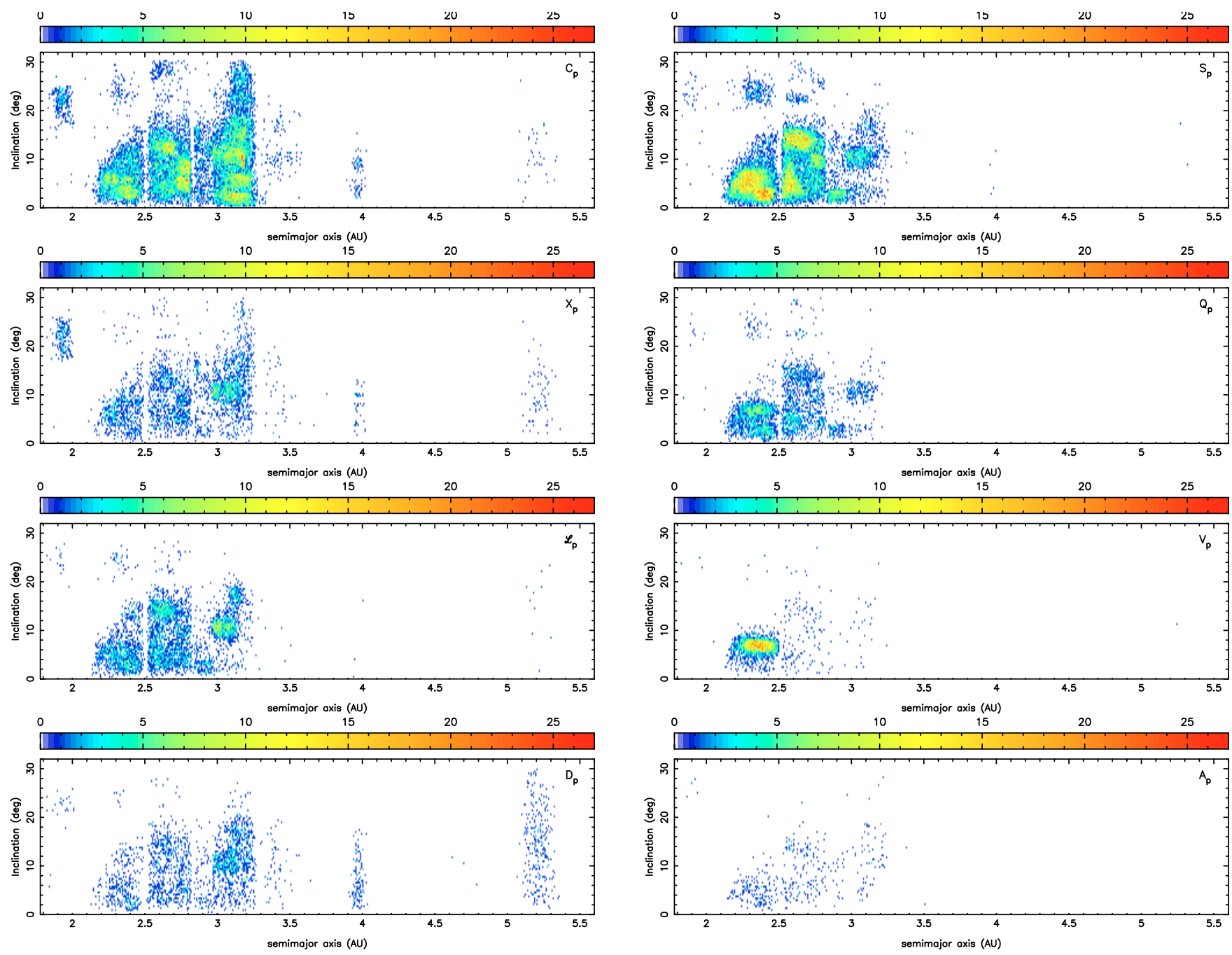

Fig. 7. Density maps for each photometric class.

select the confidence level of the classification that is adequate to their particular needs. The application of the scheme to the fourth release of the SDSS Moving Object Catalogue yields the classification of 107466 observations of 63468 asteroids with a probability over $5 \%$.

Applying of this taxonomy to meteorite spectra showed that objects with a given mineralogy can be spread through different taxonomic classes. In turn, each taxonomic class is, in general, compatible with several different meteorite classes. This exercise again shows that even in the absence of processes such as space weathering, the information concerning the mineralogy of an asteroid that can be obtained only by taxonomy is very limited.

We also used asteroids with multiple observations to verify the issue of taxonomic variability. It was found that color variations that are sufficient to lead to varying taxonomic classifications between observations of the same body are fairly common. Most of these variations may be the result of random errors and the low spectral resolution of the SDSS data, but some of the most extreme could be real and deserve further attention. Finally, the large number of classified objects allow a much more detailed and robust view of the distribution of taxonomic classes in the main belt. Most of the features highlighted in the previous section lack satisfactory explanations. Although some might be related to relatively recent events, like family formation,

some others might contain clues to processes that took place in the early stages of proto-planetary disk and may provide valuable constraints to the models of formation and evolution of the Solar System. Again, the taxonomic classification provided in this work can be instrumental in these studies.

Acknowledgements. The authors acknowledge FAPERJ and CNPq for several grants and fellowships.

\section{References}

Alvarez-Candal, A., Duffard, R., Lazzaro, D., \& Michtchenko, T. 2006, A\&A, 459, 969

Barucci, M. A., Capria, M. T., Coradini, A., \& Fulchignoni, M. 1987, Icarus, 72, 304

Bell, J. E., \& Keil, K. 1988, in Lunar and Planetary Science Conference Proceedings, ed. G. Ryder, 18, 573

Binzel, R. P., \& Xu, S. 1993, Science, 260, 186

Binzel, R. P., Xu, S., Bus, S. J., et al. 1993, Science, 262, 1541

Binzel, R. P., Harris, A. W., Bus, S. J., \& Burbine, T. H. 2001, Icarus, 151, 139

Binzel, R. P., Rivkin, A. S., Stuart, J. S., et al. 2004, Icarus, 170, 259

Binzel, R. P., Masi, G., \& Foglia, S. 2006, in BAAS, 38, 627

Binzel, R. P., Masi, G., Foglia, S., et al. 2007, in Lunar and Planetary Institute Science Conference Abstracts, 38, 1851

Bowell, E. 2009, VizieR Online Data Catalog, 1, 2001

Bowell, E., Chapman, C. R., Gradie, J. C., \& Zellner, B. 1978, Icarus, 35, 313

Britt, D. T., \& Pieters, C. M. 1989, Meteoritics, 24, 255

Britt, D. T., Tholen, D. J., Bell, J. F., \& Pieters, C. M. 1992, Icarus, 99, 153

Burbine, T. H. 2000, Ph.D. Thesis, MIT 
A\&A 510, A43 (2010)

Burbine, T. H., \& Binzel, R. P. 2002, Icarus, 159, 468

Burbine, T. H., Buchanan, P. C., Binzel, R. P., et al. 2001, Meteoritics and Planetary Science, 36, 761

Bus, S. J. 1999, Ph.D. Thesis, AA Massachusetts Institute of Technology

Bus, S. J., \& Binzel, R. P. 2002a, Icarus, 158, 146

Bus, S. J., \& Binzel, R. P. 2002b, Icarus, 158, 106

Carvano, J. M., Mothé-Diniz, T., \& Lazzaro, D. 2003, Icarus, 161, 356

Cellino, A., Bus, S. J., Doressoundiram, A., \& Lazzaro, D. 2002, Asteroids III, 633

Chapman, C. R. 1996a, Meteoritics and Planetary Science, 31, 699

Chapman, C. R. 1996b, Meteoritics and Planetary Science, 31, 699

Chapman, C. R., Morrison, D., \& Zellner, B. 1975, Icarus, 25, 104

DeMeo, F. E., Binzel, R. P., Slivan, S. M., \& Bus, S. J. 2009, Icarus, 202, 160

Duffard, R., \& Roig, F. 2009, Planet. Space Sci., 57, 229

Florczak, M., Lazzaro, D., \& Duffard, R. 2002, Icarus, 159, 178

Fukugita, M., Ichikawa, T., Gunn, J. E., et al. 1996, ApJ, 111, 1748

Gaffey, M. J., Burbine, T. H., Piatek, J. L., et al. 1993, Icarus, 106, 573

Gaffey, M. J., Cloutis, E. A., Kelley, M. S., \& Reed, K. L. 2002, Asteroids III, 183

Gradie, J. C., Chapman, C. R., \& Tedesco, E. F. 1989, in Asteroids II, ed. R. P. Binzel, T. Gehrels, \& M. S. Matthews, 316

Hapke, B. 2001, J. Geophys. Res., 106, 10039

Hiroi, T., Kanno, A., Nakamura, R., et al. 2003, in Lunar and Planetary Inst. Technical Report, Lunar and Planetary Institute Science Conference Abstracts, ed. S. Mackwell \& E. Stansbery, 34, 1425

Ivezić, Ž., Tabachnik, S., Rafikov, R., et al. 2001, ApJ, 122, 2749

Jewitt, D. C. 2002, AJ, 123, 1039

Jones, T. D., Lebofsky, L. A., Lewis, J. S., \& Marley, M. S. 1990, Icarus, 88, 172

Lazzaro, D., Michtchenko, T., Carvano, J. M., et al. 2000, Science, 288, 2033

Lazzaro, D., Angeli, C. A., Carvano, J. M., et al. 2004, Icarus, 172, 179

McCord, T. B., Adams, J. B., \& Johnson, T. V. 1970, Science, 168, 1445
McFadden, L. A., Gaffey, M. J., \& McCord, T. B. 1984, Icarus, 59, 25 McFadden, L. A., Gaffey, M. J., \& McCord, T. B. 1985, Science, 229, 160 Misra, A., \& Bus, S. J. 2008, in BAAS, 40, 40, 508

Moskovitz, N. A., Jedicke, R., Gaidos, E., et al. 2008, Icarus, 198, 77

Mothé-Diniz, T., \& Carvano, J. M. 2005, A\&A, 442, 727

Mothé-Diniz, T., \& Nesvorný, D. 2008, A\&A, 486, L9

Mothé-Diniz, T., Carvano, J. M. Á., \& Lazzaro, D. 2003, Icarus, 162, 10

Mothé-Diniz, T., Roig, F., \& Carvano, J. M. 2005, Icarus, 174, 54

Mothé-Diniz, T., Carvano, J. M., Bus, S. J., \& Burbine, T. H. 2008, Icarus, 195, 277

Neese, C. 2006, NASA Planetary Data System, EAR-A-5-DDR-TAXONOMYV5.0, 48

Nesvorný, D., Roig, F., Gladman, B., et al. 2008, Icarus, 193, 85

Parker, A., Ivezić, Ž., Jurić, M., et al. 2008, Icarus, 198, 138

O’Brien, D. P., Morbidelli, A., \& Bottke, W. F. 2007, Icarus, 191, 434

Pieters, C. M., \& Hiroi, T. 2004, in Lunar and Planetary Inst. Technical Report, Lunar and Planetary Institute Science Conference Abstracts, ed. S. Mackwell, \& E. Stansbery, 35, 1720

Rivkin, A. S., Howell, E. S., Britt, D. T., et al. 1995, Icarus, 117, 90

Rivkin, A. S., Howell, E. S., Lebofsky, L. A., \& Britt, D. T. 2000, Icarus, 145, 351

Roig, F., \& Gil-Hutton, R. 2006, Icarus, 183, 411

Roig, F., Nesvorný, D., Gil-Hutton, R., \& Lazzaro, D. 2008, Icarus, 194, 125

Sasaki, S., Nakamura, K., Hamabe, Y., et al. 2001, Nature, 410, 555

Sunshine, J. M., Pieters, C. M., \& Pratt, S. F. 1990, J. Geophys. Res., 95, 6955

Sunshine, J. M., Bus, S. J., Corrigan, C. M., \& Burbine, T. H. 2007, Meteoritics and Planetary Science, 42, 155

Szabó, G. M., Ivezić, Ž., Jurić, M., \& Kiss, L. L. 2004, MNRAS, 348, 987

Tedesco, E. F., Williams, J. G., Matson, D. L., et al. 1989, AJ, 97, 580

Tholen, D. J. 1984, Ph.D. Thesis, AA Arizona Univ., Tucson

Vilas, F., \& Gaffey, M. J. 1989, Science, 246, 790 